\title{
Human Immunodefieciency Virus: Pharmacogenetics of Antiretroviral Treatment
}

\author{
Susana A Pesoa ${ }^{1}$, Cristian A Galván Bioch ${ }^{1,2}$, Dante M Beltramo ${ }^{2,3,4 *}$ and Néstor W Soria ${ }^{1,2 *}$ \\ ${ }^{1}$ Laboratorio de Análisis Clínicos Especializados (LACE), Córdoba, Argentina \\ ${ }^{2}$ Cátedra de Biotecnología, Facultad de Ciencias Químicas, Universidad Católica de Córdoba, Córdoba, Argentina \\ ${ }^{3}$ Centro de Excelencia en Productos y Procesos de Córdoba (CEPROCOR), Santa María de Punilla, Córdoba, Argentina \\ ${ }^{4}$ Consejo Nacional de Investigaciones Científicas y Técnicas (CONICET)
}

\begin{abstract}
The introduction of highly active antiretroviral therapy as standard of care has considerably enhanced the life expectancy among HIV-infected individuals. Although this combination of drugs virtually suppresses viral replication the therapeutic effect may be limited by differing rates of adverse events and responses in terms of efficacy and toxicity. These differences arise from complex interactions between biological and environmental factors. Pharmacogenetic studies are contributing to our understanding of the inter-individual differences in the response to antiretroviral drugs. Several studies have provided a relevant number of associations between human genetic variants and predisposition to adverse events and for some antiretroviral drugs clear and causal genotype-phenotype correlation has been established. These findings make the idea of personalized medicine in this field increasingly attractive. We will discuss here current achievements on pharmacogenetics of HIV treatment with special emphasis on the genetic polymorphisms underlying toxic effects and/or those already implemented in the clinical setting.
\end{abstract}

\section{Introduction}

\section{General considerations on pharmacogenetics}

Human immunodeficiency virus (HIV) infection is recognized as one of the most serious contemporary pandemics. The introduction of highly active antiretroviral therapy (HAART) as standard of care has changed the natural history of HIV infection into a controllable chronic disease requiring long-term antiretroviral (ARV) treatment. Effective HAART and the availability of genetic screening of patient virus data have conducted to sustained viral suppression and higher life expectancy in infected patients [1-3]. However, response to HAART is often limited by the occurrence of toxicity or by the emergence of drug resistance. Cohort studies from the United States and Europe indicate that around $20-30 \%$ of patients initiating therapy discontinue treatment in the first year [4].

It is important to remark here that overall, adverse drug reactions (ADRs) are regarded as an important public health problem, as they can cause serious diseases and even be life-threatening. They are defined by the World Health Organization as noxious and unintended responses to drugs at normal doses. The classical pharmacological classification of ADRs by Rawlins [5] distinguishes two types of reactions: type A, which is dose-dependent and predictable and type $\mathrm{B}$, which are not dose-dependent and are unpredictable. Type B reactions comprise approximately $10-15 \%$ of all ADRs and include hypersensitivity drug reactions (HSRs). It has even been estimated that adverse drug reactions, many of which is due to variations in the pharmacokinetic of drugs, are responsible for around 7\% of all hospital admissions [6].

As mentioned earlier, in the HIV infection setting, antiretroviral treatment is characterized by differing rates of responses and adverse events. Numerous studies report that the administration of standard doses of most antiretroviral drugs results in significant variations in plasma drug concentrations among different individuals, influencing antiviral activity as well as incidence of drug-related toxicities. These differences arise from complex interactions between biological and environmental factors, which include variations in virus biology and host physiology, concomitant medications, drug compliance, underlying diseases, socioeconomic disparities, regional preferences in treatment standards and genetic diversity affecting drug metabolism and the expression of drug targets [7]. In this scenario, genetic associations highlighting differences in the response to HIV infection and treatment have significantly furthered our understanding of the pathogenesis, pharmacokinetics and pharmacodynamics of antiretroviral drug action and toxicities and HIV disease itself. In fact, HIV-infected patient's response differs genetically with regard to both the virus and the ARV drugs [8]. These interindividual differences in the pharmacokinetics of drugs represent a major clinical problem since plasma levels of drugs are poorly predictable. Pharmacogenomic studies have provided important results allowing significant improvements in the treatment of a number of conditions [9-11]. In this context, it is very well known that the overall pharmacologic effects of drugs are typically not monogenic traits; rather, effects are determined by the interaction of several genes encoding different proteins involved in different pathways of drug metabolism, disposition and effects. A relevant number of associations between human genetic variants and predisposition to adverse events have been described and for some antiretroviral drugs a clear and causal genotype-phenotype correlation has been established $[7,12]$.

*Corresponding authors: Dr. Néstor W. Soria, Laboratorio de Análisis Clínicos Especializados (LACE), Avenida Vélez Sarsfield 528, (5000) Córdoba, Argentina Tel/Fax: 54-351-4246666; E-mail: nes_soria@yahoo.com.ar

Dr. Dante M. Beltramo, Laboratorio de Análisis Clínicos Especializados (LACE) Avenida Vélez Sarsfield 528, (5000) Córdoba, Argentina, Tel/Fax: 54-351-4246666; E-mail: dbeltramo@ceprocor.uncor.edu

Received October 14, 2011; Accepted November 22, 2011; Published November 25, 2011

Citation: Pesoa SA, Galván Bioch CA, Beltramo DM, Soria NW (2011) Human Immunodefieciency Virus: Pharmacogenetics of Antiretroviral Treatment. J Pharmacogenom Pharmacoproteomics S6:002. doi:10.4172/2153-0645.S6-002

Copyright: (C) 2011 Pesoa SA, et al. This is an open-access article distributed under the terms of the Creative Commons Attribution License, which permits unrestricted use, distribution, and reproduction in any medium, provided the original author and source are credited. 
Citation: Pesoa SA, Galván Bioch CA, Beltramo DM, Soria NW (2011) Human Immunodefieciency Virus: Pharmacogenetics of Antiretroviral Treatment. J Pharmacogenom Pharmacoproteomics S6:002. doi:10.4172/2153-0645.S6-002

The biomedical discipline analyzing the effects of polymorphisms within human genes on drug metabolism and drug toxicity is the Pharmacogenetics. This field has been pursued in HIV therapeutics because of the prevalence of toxicity, the long-term nature of treatment, and the inherent complexity of multidrug therapy that could benefit from predictive tools to identify the drug combination most likely to be tolerated and effective [13]. In this scenario, it is reasonable to hypothesize that pharmacogenetic studies coupled with molecular characterization of the HIV may help moving towards improved personalized therapy. It should also be considered that while basal expression of drug metabolizing enzymes or transporters may be unaffected by mutations, the degree of inducibility by other drugs co-administered with antiretroviral treatment (ART) like rifampicin for example, may vary when polymorphisms affect gene regulatory motifs such as promoter or nuclear factors (i.e. PXR (pregnane X receptor) and CAR (constitutive androstane receptor), which are also polymorphic) [14].

To date, more than twenty antiretroviral drugs have been approved by the US Food and Drug Administration (FDA) for the treatment of HIV. As it is depicted in Figure 1 these agents target relevant molecules in the HIV life cycle aiming to prevent virus entry and /or replication. According to their mechanism of action these compounds can be categorized as: (a) nucleoside/nucleotide reverse transcriptase inhibitors (NRTIs or NtRTIs), (b) non-nucleoside reverse transcriptase inhibitors (NNRTIs), (c) protease inhibitors (PIs), (d), integrase inhibitors, (e) fusion/entry inhibitors.

Research focused on the discovery and development of innovative agents for HIV treatment is especially active with entry inhibitors receiving the most of attention in the field. HIV entry is a three-step process. The C-C Chemokine Receptor Type 5 (CCR5) antagonist Maraviroc targets the second step [15] and the fusion inhibitor Enfuvirtide acts at the third step [16]. Interestingly, BMS-663068 would be the first drug to target the initial step (http://cenblog.org/the- haystack/tag/bms-663068/). Its active form, BMS-626529, works by binding to the HIV-1 envelope glycoprotein gp120, thereby interfering with its attachment to the CD4 receptor. On the contrary, TNX-355, is a monoclonal antibody that inhibits HIV attachment by binding to host cell CD4 receptors [17]. Chemokine antagonists inhibit the middle step by preventing HIV from binding with one of two co-receptors CCR5 or C-X-C Chemokine Receptor Type 4 (CXCR4). Several CCR5 antagonists are being studied such as, UK-427-857 [15], SCH-D [18], and the monoclonal antibody PRO-140 [19]. The agent called vMIP-II appears to block multiple co-receptors [20] and this approach could prevent HIV from switching back and forth between co-receptors, however, given that chemokines perform multiple functions, agents aiming to inhibit their activity could potentially produce serious side effects. SJ-3366 inhibits entry after that HIV attaches to CD4 cells [21], but its exact mechanism of action is unknown. Finally, other compounds like FP-21399 inhibit the fusion step [22] and focus on ARV research also includes next-generation NRTI such as Festinavir.

International ARV treatment guidelines currently recommend a daily combination of three or more antiretroviral drugs for the treatment of immunocompromised HIV infected patients. The combination treatment referred as HAART is composed of at least three drugs from two different classes, i.e. two NRTIs plus one NNRTI or two NRTIs plus one Protease Inhibitor (PI). Although HAART cannot eradicate HIV infection, it can prevent progression to acquired immune deficiency syndrome (AIDS) by suppressing the level of viral replication, thus helping to restore the immune system [23].

Host genetic factors influencing efficacy and toxicity of ARV drugs have been assessed in a number of prospective HIV clinical trials and cohort studies. The genetic variants analyzed were single nucleotide polymorphisms (SNPs) and haplotypes (combinations of SNPs that are inherited together), which can be phenotypically associated with altered enzymatic activity and pharmacokinetics and pharmacodynamics of ARV agents. In addition, these studies included: HSR syndromes,

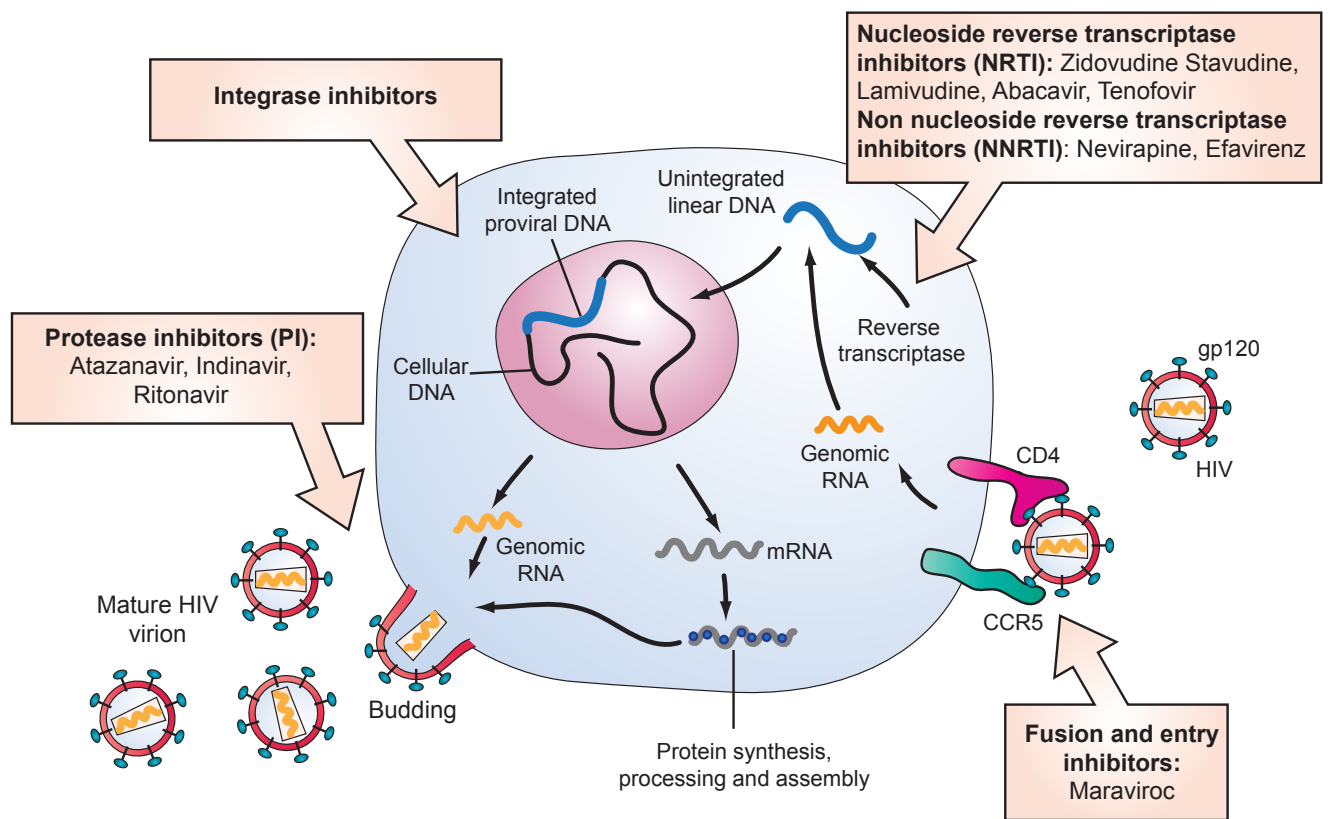

Figure 1: Human Immunodeficiency Virus life cycle: Targets for antiretroviral intervention. Approved drugs are categorized according to their mechanism of action. Only drugs potentially associated to pharmacogenetic markers of efficacy and/or toxicity are depicted. 
Citation: Pesoa SA, Galván Bioch CA, Beltramo DM, Soria NW (2011) Human Immunodefieciency Virus: Pharmacogenetics of Antiretroviral Treatment. J Pharmacogenom Pharmacoproteomics S6:002. doi:10.4172/2153-0645.S6-002

hepatotoxicity, central nervous system side effects, hyperbilirubinemia, peripheral neuropathy, lipodystrophy, hyperlipidaemia, pancreatitis and renal toxicity.

It is important to note, that numerous reports describe differences in the distribution of genetic polymorphisms affecting HAART treatment among diverse ethnic groups. Endeavors such as the Internal Frequency optional HapMap Project have provided insight into not only the quantity of polymorphism but also the variability of polymorphism frequency between world populations. In the context of HIV treatment, it has been reported for example that drug exposure to the PI Saquinavir is significantly increased in Thai patients compared with Caucasians [24]. Emerging data also show that the NNRTI Efavirenz has a longer elimination half-life in Black Africans than in Caucasians [25]. In other study, it was seen that patients from Thailand and South Africa had lower clearances of Efavirenz than those from Europe or the Americas [26]. Genetic variability in drug metabolizing enzymes (e.g. cytochrome P450, (CYP450); glucuronyl transferase, GT) or drug transporters, e.g. ABCB1 (P-glycoprotein; MDR1), ABCC1 (MRP1) and ABCC2 (MRP2), prevalent at differing frequencies across ethnic groups, probably explains some of the differences among populations; although as mentioned above other factors such as body weight may also contribute to these findings [27]. Furthermore, it is important to remark that although the frequency of most described functional polymorphisms have been assessed in multiple populations and a number of comparisons have been performed, data are far from being comprehensive. For this reason, an important step prior to translating pharmacogenetic testing to the clinical practice is to perform studies in order to depict the genetic profile of the local population. In this perspective, the lack of information for the Argentinean population (except for CCR5 [28,29]; prompted us to investigate the allelic frequencies of the CYP2B6 (rs3745274), CCR5 (rs333), HLA complex P5 (HCP5) (rs2395029) polymorphisms recognized as predictive markers for treatment efficacy with Efavirenz, Maraviroc and Abacavir, respectively. The study was conducted in Argentineans subjects from the central area of our country known to have an important

\begin{tabular}{|c|c|c|c|}
\hline CLASIFICATION & DRUGS & GENES - RS & ADVERSE EVENTS \\
\hline \multirow{2}{*}{ Nuclec } & Zidovudine & $\begin{array}{c}\text { ABCC4 (MRP4) } \\
\text { A1203A - rs11568695 }\end{array}$ & $\begin{array}{l}\text { Anemia, neutropenia } \\
\text { Fatigue, malaise, headache } \\
\text { Nausea, vomiting } \\
\text { Myalgia, myopathy }\end{array}$ \\
\hline & Stavudine & $\begin{array}{c}\text { POLG (Mit DNA) } \\
\text { R964C and E1143G }\end{array}$ & $\begin{array}{l}\text { Peripheral neuropathy } \\
\text { Pancreatitis } \\
\text { Dyslipidaemia } \\
\text { Diarrhea }\end{array}$ \\
\hline Reverse & Lamivudine & $\begin{array}{c}\text { ABCC2 (MRP2) } \\
\text { C421A-Q141K - rs2231142 } \\
\text { Q126X - rs725522713 } \\
\text { V12M - rs2231137 } \\
\text { MRP4 } \\
\text { 3' UTR - rs3742106 }\end{array}$ & Headache, dry mouth \\
\hline \multirow{2}{*}{ Inhibitors } & Abacavir & $\begin{array}{c}\text { HLA-B*5701 - rs3093726 } \\
\text { HCP-5 - rs2395029 }\end{array}$ & $\begin{array}{l}\text { Hypersensitivity syndrome (HSR) } \\
\text { Rash, fever, myalgia, malaise, nausea, } \\
\text { vomiting,diarrhea }\end{array}$ \\
\hline & Tenofovir & $\begin{array}{c}\text { ABCC4 (MRP4) } \\
\text { K1116K - rs1751034 }\end{array}$ & $\begin{array}{l}\text { Asthenia } \\
\text { Acute renal insufficiency } \\
\text { Proximal Tubulopathy } \\
\text { Fanconi syndrome }\end{array}$ \\
\hline \multirow{2}{*}{$\begin{array}{l}\text { Non Nucleoside } \\
\text { Reverse } \\
\text { Transcriptase } \\
\text { Inhibitors }\end{array}$} & Nevirapine & $\begin{array}{c}\text { ABCB1 } \\
\text { I1143| - rs1045642 } \\
\text { CYP2B6 } \\
\text { Q172H - rs3745274 } \\
\text { I328T - rs28399499 }\end{array}$ & $\begin{array}{l}\text { Hypersensitivity syndrome (HSR) } \\
\text { Skin rash } \\
\text { Elevations in liver function tests, Hepatitis, liver failure }\end{array}$ \\
\hline & Efavirenz & $\begin{array}{c}\text { CYP2B6 } \\
\text { Q172H - rs3745274 } \\
\text { I328T - rs28399499 } \\
\text { ABCB1 (MDR1) } \\
\text { |1143| - rs1045642 }\end{array}$ & Peripheral Neuropathy \\
\hline \multirow{3}{*}{$\begin{array}{l}\text { Protease } \\
\text { Inhibitors }\end{array}$} & Atazanavir & $\begin{array}{c}\text { UGT1A1 } \\
\text { UGT1A1*28- (rs8175347) }\end{array}$ & $\begin{array}{l}\text { Hyperbilirubinemia, jaundice } \\
\text { Elevations in liver function tests, PR interval } \\
\text { prolongation }\end{array}$ \\
\hline & Indinavir & $\begin{array}{c}\text { UGT1A1 } \\
\text { UGT1A1*28 - rs8175347 } \\
\text { CYP3A4/ CYP3A5 } \\
\text { CYP3A4*1B /CYP3A5*1 }\end{array}$ & $\begin{array}{l}\text { Hyperbilirubinemia, jaundice } \\
\text { Nephrolithiasis, flank pain }\end{array}$ \\
\hline & Ritonavir & ABCA1, APOA5, APOC 3, APOE, CETP & Dyslipidaemia \\
\hline Entry Inhibitor & Maraviroc & $\begin{array}{c}\text { CCR5 } \\
\Delta 32-\text { rs333 }\end{array}$ & $\begin{array}{l}\text { Diarrhea, nausea } \\
\text { Upper respiratory tract infections, cough. Joint, muscle } \\
\text { pain }\end{array}$ \\
\hline
\end{tabular}

Table 1: Summary of genetic variants associated to antiretroviral pharmacokinetics, toxicity and/or efficacy in HIV treatment. 
contribution of European immigration of Italian and Spanish origin to its genetic pool. Interestingly, the frequency of the CYP2B6 rs3745274 mutation observed in our population was higher than the observed in Europeans pointing out the relevance of this study before starting a protocol involving Efavirenz as we will discuss below. In the case of CCR5 gene, therapeutic target of Maraviroc, (See Figure 1 and Table 1 ), the frequency of the $\Delta 32$ variant (rs333) [30] in our population was similar to the reported for the Amerindian Chiriguanos [28], for Jamaicans [31] and Italians [32], but higher than the reported for Argentinean subjects from Chaco (with Guarani and Hispanic genetic background) [29]. Of note, the mutant allele of CCR5 is found at high frequency in Caucasians but is absent in the Chinese [31] and black populations. Finally, the allele frequency for HCP5 (rs2395029) in our population was very similar to Italian, Spanish, Mexican, Chinese and Japanese people. It is important to remark that the study of individuals from different geographic regions of Argentina, receiving distinct immigration waves, will further elucidate the relative contribution of diverse polymorphic variants to the genetic pool of interest in pharmacogenetics in our country [33].

In conclusion, the ultimate goal in pharmacogenetics is to define robust markers that can be used in the clinical setting to support the individualized treatment selection. It has been already shown that individualized drug therapy based on the presence of genetic polymorphisms can result in an important reduction of adverse side effects in the clinic $[34,35]$. However, despite the plethora of genetic information the true number of established genetic markers ready for integration in clinical practice is remarkably small and their usefulness may be geographically limited. Although many initial associations are intriguing they are really only tentative until replicated by multiple studies performed by independent groups. Commonly published polymorphisms often suffer from contradictory results making their role in the clinic unclear.

\section{Towards individualized HAART: The pharmacogenetic portfolio}

The complex pharmacokinetic properties of individual ARV in HAART regimens stimulated numerous studies to explore the pharmacogenetic determinants involved, as clinicians were aware of the viral suppression potential of HAART but also considered the unpredictable nature of these drug interactions. The studies considered first, the drug metabolizing capacity of the isoforms of CYP450 oxidative enzymes and their contribution to drug interactions. In the case of the NNRTIs, Nevirapine and Efavirenz, their inductive characteristics created concern for the enhanced metabolism of concurrently administered medications, while the use of PIs such as Atazanavir or Indinavir, with inhibitory effects on CYP3A, led to a specially challenging scenario. In contrast, there is very little involvement of CYP450 enzymes in NRTI metabolism. For this reason, in this category of agents most studies have focused in genes involved in drug transport and HSR.

\section{Nucleoside reverse transcriptase inhibitors}

NRTIs are a class of antiretroviral compounds whose chemical structure represents a modified version of a natural nucleoside. These agents, after intracellular phosphorylation suppress HIV replication by interfering with the reverse transcriptase enzyme [36,37]. In general, NRTI adverse effects are associated with lactic acidosis, hepatic steatosis and body fat redistribution (lipodystrophy). Additional evidence relates NRTI agents to disruption of mitochondrial function and oxidative stress and peripheral neuropathy [38]. As mentioned above most studies on pharmacogenetic determinants of NRTIs toxicity have focused on HSR syndromes and the HLA system. Additionally, genes involved in drug transport, like multidrug resistance proteins (MRP), polymorphisms in mitochondrial genome and factor Tumor Necrosis Factor alpha (TNF-alpha) have also been considered regarding NRTI toxicity.

Abacavir: Abacavir, a guanosine nucleoside analog is a potent antiretroviral agent that competitively inhibits the reverse transcriptase of HIV-1 leading to chain synthesis termination. About $5 \%-8 \%$ of HIV infected individuals who initiate Abacavir therapy develop a HSR characterized by fever, rash, abdominal complaints, lethargy and acute respiratory symptoms, within the first 4-6 weeks of treatment; the reaction generally improves within $24 \mathrm{~h}$ following drug discontinuation. However, rechallenge with Abacavir may result in severe recurrence of symptoms, with increased risk of life-threatening complications and death [39]. The underlying immunologic mechanism of Abacavir HRS is an HLA-B5701-restricted immune response to the NRTI drug [40]. The strong association between Abacavir HSR and HLA-B ${ }^{\star} 5701$ has been demonstrated in both observational and blinded randomized clinical trials in racially diverse populations and represents the best example of the clinical utility of pharmacogenetic screening in HIV medicine. Indeed, the use of HLA-B ${ }^{\star} 5701$ as a screening test is one of the first examples of pharmacogenetics being feasibly implemented in the clinical setting to prevent a previously unpredictable toxicity of a specific drug [41].

Based on evidence from PREDICT-1 [42] and other studies [43], most national and international HIV treatment guidelines, including those of the International AIDS Society (IAS) and US Department of Health and Human Services (DHHS), have recommended genotyping for HLA-B ${ }^{\star} 5701$ into routine clinical practice as the standard of care for all patients before prescribing an Abacavir containing regimen.

It is important to note here, that a prerequisite for pharmacogenetics to be applied in the clinical practice is the development of fast, reliable, and cost-effective techniques for the routine genotyping of patients. For this reason, another genetic marker proposed as surrogate of the HLA-B ${ }^{\star} 5701$ allele and Abacavir HSR is a polymorphism located within the HLA complex P5 gene, HCP5 rs2395029. This polymorphism was found to be in high linkage disequilibrium with HLA-B ${ }^{\star} 5701$ by several studies [44], allowing the clinical use of the former marker as an alternative to HLA typing $[44,45]$.

In conclusion, the major short term adverse effect of Abacavir, the HSR, can be largely avoided by excluding it from the cocktail in patients positive for HLA-B`5701 or HCP5 rs2395029.

Zidovudine / Lamivudine: Zidovudine, an analog of thymidine, was the first approved treatment for HIV, representing a major breakthrough in AIDS therapy in the 1990s since significantly altered the course of the infection. Lamivudine, on the other hand, is an analogue of cytidine often given in combination with Zidovudine, with which it is highly synergistic. Lamivudine treatment has been shown to restore Zidovudine sensitivity of previously resistant HIV. It has been shown that intracellular concentration of Zidovudine and other NRTIs are mainly determined by the activities of $\mathrm{P}$ glycoprotein (P-gp) a cell membrane efflux pump and the multidrug resistance associated proteins (MDR) MRP2 and MRP4. Anderson et al. [46] investigated the relationships among Lamivudine-triphosphate, and ZDV-triphosphate pharmacokinetics and pharmacodynamics with 
polymorphisms in MDR1, MRP2 and MRP4. Two polymorphisms at MRP-4 might have clinical relevance in HIV therapy. The T4131G change has been associated with higher intracellular concentrations of Lamivudine-triphosphate. In addition, other polymorphisms at MRP2 may also contribute to increased plasma levels of Lamivudine (See Table 1). On the other hand, the G3724A change seems to result in more elevated Zidovudine-triphosphate intracellular levels [46]. At present, it is unclear in what extent the variability observed in the response to these drugs could be explained by genetic variability at the MRP transporters.

Tenofovir: Tenofovir (TDF) is a potent and well tolerated oncedaily NRTI; however, its use has been associated with nephrotoxicity, produced by accumulation of the drug in renal proximal tubules. Renal injury in patients receiving the drug has also been associated with the presence of co-morbidities and with advanced HIV infection [47]. Although further studies are needed to confirm this relationship, the mechanism of nephrotoxicity could be linked to an impaired active Tenofovir efflux from the renal proximal tubular cells by the MRP2/MRP4 transporter [48]. In this context, it has been reported that renal tubulopathy was significantly associated with a single $\mathrm{G}>\mathrm{A}$ substitution at position 1249 of ATP-binding cassette, sub-family C, member 2 (ABCC2) gene. After controlling for age, sex, and duration of HIV infection, ABCC2 haplotypes were significantly associated with the onset of renal proximal tubulopathy. CATC motif appeared to be a predisposing haplotype, since it was found in a high percentage of patients presenting this side effect [49]. Interestingly, although these results point towards a putative role for MRP2 polymorphisms in the renal toxicity, it is important to note that Tenofovir is a substrate for MRP4, but not for MRP2 [50]. More recently, a novel relation between ABCC4 3463A $>$ G genotype and renal toxicity has been reported. This study shows Tenofovir concentrations $35 \%$ higher in carriers of the $3463 \mathrm{G}$ variant [48]. Thus, the mechanisms underlying these findings remain elusive and further investigations in this field are needed.

Stavudine: In general terms, NRTIs agents, although relatively specific for viral reverse transcriptase, also have varying affinities for human mitochondrial DNA (mtDNA) polymerase- $\gamma$ (POLG), the primary enzyme responsible for mtDNA replication; this has been particularly reported in the case of Stavudine, a thymidine analogue. The mtDNA POLG hypothesis holds that inhibition of mtDNA POLG by NRTIs disrupts normal mitochondrial replication, leading to a cascade of events that culminates in impaired oxidative phosphorylation due to mitochondrial dysfunction, increased production of free radicals and oxidative stress, and ultimately tissue injury and symptomatic toxicity. Details for this cascade have not been fully characterized, but the recent report of a novel, functional mtDNA POLG mutation being associated with NRTI-associated lactic acidosis supports the POLG inhibition hypothesis [51,52]. Several lines of evidence implicate mitochondrial injury in NRTI toxicities, including peripheral neuropathy $[38,53]$. Recently, Stavudine-induced mitochondrial toxicity was associated to POLG mutations R964C and E1143G. Moreover, it has been shown that carriers of the mutant Mip1p (R964C) and possibly Mip1p (E1143G) are more sensitive to Stavudine; furthermore it has been reported that Stavudine has the potential to cause mitochondrial toxicity in heterozygous subjects harboring recessive mutations [54].

Additionally, studies on the genetic factors that might influence the susceptibility to NRTI-associated peripheral neuropathy, found that a high percentage of patients receiving the guanosine analogue Didanosine and/or Stavudine who developed peripheral neuropathy had a particular mitochondrial DNA genetic configuration, the so called haplogroup T. Interestingly, among non-Hispanic whites randomized to receive Didanosine and/or Stavudine, mitochondrial haplogroup $\mathrm{T}$, a common European mitochondrial haplogroup, independently predicted neuropathy during the study treatment. Further exploration of the effect of non-synonymous mtDNA polymorphisms underlying haplogroup- $T$ revealed that the variant $4917 \mathrm{G}$ was significantly associated with peripheral neuropathy in whites [38]. Further studies are needed to fully identify relationships between host genetic variants and peripheral neuropathy associated to HIV treatment.

At last, a relevant proportion of patients exposed to some NRTIs, particularly the tymidine analogues Stavudine and to lesser extent Zidovudine, may develop peripheral lipoatrophy [55]. Lipoatrophy is characterized by peripheral fat loss of gradual onset manifested as facial thinning and as thinning of extremities and buttocks. The pathogenesis of NRTI-associated lipoatrophy is multi-factorial and genetic factors may be associated with an increased risk of developing peripheral fat wasting since this condition does not occur in all treated patients and there is a very large interindividual variability in the emergence and severity of the symptoms [55]. Tumor necrosis factor (TNF)-alpha, a cytokine promoting adipocyte apoptosis, has many actions that are consistent with the features of lipodystrophy and shows high levels of expression in adipose tissue in HIV-infected patients Additional genes involved in lipid metabolism and apoptosis could play a role in the development of lipoatrophy. These include apolipoproteins (APO), adrenergic receptors and Fas and its ligand (FasL). In conclusion, although genetic polymorphisms of genes involved in apoptosis and adipocyte metabolism have been associated with the risk of developing lipoatrophy, to date, genetic prediction of fat loss is not possible. More than $50 \%$ of patients receiving HAART may develop either metabolic or morphological abnormalities or both, however further investigation is needed in the field to fully understand the pathogenesis of these syndromes $[55,56]$.

\section{Non nucleoside reverse transcriptase inhibitors}

Non-nucleoside reverse transcriptase inhibitors are a class of antiretroviral drugs that act as noncompetitive inhibitors of the HIV reverse transcriptase. Licensed NNRTIs include Efavirenz, Nevirapine and Etravirine (Figure 1). Most studies on pharmacogenetic determinants of NNRTI disposition, efficacy and toxicity have focused on genes involved in metabolism, i.e. CYP450 enzymatic complex which is the main metabolizer involved in the liver clearance of NNRTI. These isoenzymes exhibit genetic polymorphisms resulting in enzymes with reduced expression and activity in human liver microsomes. As a consequence, there is a considerable interindividual variability in NNRTI metabolism and disposition.

In addition to cytochromes, genes encoding drug transporters and genetic polymorphisms of the HLA system have also been considered in the pharmacogenetic studies of NNRTI. In this context, both oral absorption and tissue penetration of NNRTIs are affected by the drug transporter P-gp, encoded by the gene multidrug resistance protein 1 (MDR1). Polymorphisms in the MDR1 gene associate with differences in P-gp activity and, thus, in drug disposition. For this reason, the association between allelic variants of MDR1 gene and NNRTI plasma concentrations has also been studied comprehensively [2].

Nevirapine: Nevirapine-based antiretroviral combinations are first-line regimens for HIV-1 infection in most resource-poor settings, owing to its relatively low cost, manageable pill burden and excellent 
efficacy. However, the use of Nevirapine is limited by two main factors: an immune-mediated HSR that manifests as hepatotoxicity, fever and/ or skin rash; and a fragile genetic barrier to the development of drug resistance. The HSR takes place in about $5 \%$ of patients; fatal cases of this kind of reaction have also been reported. Most of these side effects present one to six weeks after initiating treatment [7]. Interestingly, the rate of this reaction is CD4 dependent, with low CD4 cell count being protective, which suggests a CD4 $\mathrm{T}$ cell-dependent immune response to Nevirapine-associated antigens and the participation of HLA Class II alleles. However, an association as stringent as that described for Abacavir has not been found so far. Indeed, the allele HLA-DRB $1^{\star} 0101$ has been associated with cutaneous reactions, but not with hypersensitivity to the drug [12]. Further investigations are necessary to delineate the exact role of the HLA and other regions of the genome in Nevirapine toxicity.

It is important to note that less severe side effects are relatively common with this drug. Isolated skin rash presents in 15 to $20 \%$ of patients, but easily subsides with interruption of therapy. Nevirapine is metabolized primarily by the CYP3A4 and CYP2B6 enzymes into its major metabolites 2-hydroxynevirapine and 3-hydroxynevirapine, respectively. The G516T SNP in CYP2B6 (CYP2B6*6) has been reported to have a major impact on the pharmacokinetics and pharmacodynamics of Efavirenz [57]. Some studies have also shown that G516T is associated with Nevirapine plasma concentration [58]. Recently, it has been reported that heterozygosity for the T983C SNP was associated with significantly higher Nevirapine plasma levels in black patients. Previous studies have found body weight, ethnicity, gender and underlying liver disease to be important in explaining Nevirapine pharmacokinetics, however in most studies body weight was the only significant demographic factor influencing clearance [59].

Finally, other set of studies have examined the relationship between exposure to Nevirapine and virological response. The risk of virological failure in patients who receive Nevirapine-based antiretroviral therapy increases 5-fold when Nevirapine plasma concentrations are 3.0 $\mathrm{mg} / \mathrm{L}$ compared with patients with higher concentrations. As a result, a trough concentration target of $3.0 \mathrm{mg} / \mathrm{L}$ has been proposed as a minimum effective concentration [59].

Efavirenz: At present, Efavirenz is one of the ARV most commonly used. The drug is generally safe, but central nervous system complaints are common, especially during the first 3 to 4 weeks of treatment. Efavirenz is metabolized by CYP2B6 in the liver. Individual carriers, and particularly homozygous, for the allele CYP2B6*6 (SNP G516T) eliminate Efavirenz at a lower rate and present higher plasma levels of the drug [60]. As a result, neuropsychological toxicity is especially prevalent, intense, and prolonged in these persons. Another consequence of decreased metabolism of Efavirenz is the persistence of the drug in plasma after treatment interruption, which potentially increases the risk for selection of drug-resistant HIV strains. Indeed, the CYP2B6 516 TT genotype or a prolonged measured elimination half-life may predict increased risk of developing drug resistance among patients who discontinue Efavirenz-containing regimens. As previously mentioned, Efavirenz has a long plasma half-life but a relatively low genetic barrier to HIV-1 resistance. Selective expansion of Efavirenz-resistant HIV-1 in such individuals may compromise the efficacy of NNRTIs, such as Efavirenz and Nevirapine, in subsequent regimens. This has implications for strategies to safely discontinue ARV regimens while avoiding the emergence of drug resistance [57].

In conclusion, prospective testing for CYP2B6 alleles may help to select the appropriate Efavirenz doses in HIV infected individuals to avoid toxicity and drug resistance [60].

\section{Protease Inhibitors (PI)}

Protease inhibitors ARV agents hinder HIV-1 replication by inhibiting the HIV-1 protease activity. PIs are mostly metabolized by CYP3A, particularly CYP3A4. Interestingly, PIs are not only substrate but also inhibitors of CYP3A; Ritonavir for example is a very potent CYP3A inhibitor and is therefore used as a booster to increase the plasma exposure of other PIs. As a result, the impact of CYP3A polymorphisms on PI disposition is difficult to predict. In particular, CYP3A4 has received increasing attention as a candidate for pharmacogenetic investigation after the discovery of CYP3A $4{ }^{\star} 20$, the first allele which has no functional activity. Other PIs, such as Atazanavir and Indinavir are also metabolized via CYP3A, although these drugs can also be substrates for uridine diphosphate-glucuronosyltransferase $1 \mathrm{~A} 1$ (UGT1A1). The CYP3A4 ${ }^{\star} 20$ allele may have an important impact on the clinical use of these and other PIs that are substrate of CYP3A enzyme. Several recent studies have shown that CYP3A5 ${ }^{\star} 1$ also contributes to the disposition of PIs. A recent report by Mouly et al. [61] has shown that CYP3A $5^{\star} 1$ genotype is associated with increased Saquinavir clearance, which was twice as high as among the individuals carrying the CYP3A5*3 allele [62].

Atazanavir: Atazanavir, provokes a mild increase in plasma levels of unconjugated bilirubin in most patients. This side effect is provoked by competitive inhibition of UGT1A1, the microsomal enzyme in charge of glucuronidation, i.e., transformation of unconjugated bilirubin into conjugated bilirubin, which allows bilirubin excretion into the bile. The rise is inconsequential, and only a minority of patients has to discontinue therapy because of overt jaundice. The presence of allele UGT1A1*28, a gene variant associated with decreased enzyme activity, is strongly associated with the occurrence of jaundice in patients who take the medication $[46,63]$. Other alleles of the gene that encodes UGT1A1 are also probably involved [63].

Indinavir: Indinavir has been one of the preferred PI included in HAART. At present, even if not recommended as initial therapy, this agent is still used since, compared to other PIs, Indinavir exhibits a high penetration into viral reservoirs such as genital compartments and central nervous system [64]. It has been reported that Indinavir pattern of distribution associates to the enhanced outcomes observed in neurological complications related to HIV. The pharmacokinetics of Indinavir is characterized by high maximal concentrations leading to potential toxicity, notably nephrolithiasis and low minimum concentrations with respect to the $95 \%$ inhibitory concentration of the virus [65]. Efficacy as well as adverse events has been related to Indinavir plasma concentrations. The low residual concentrations result from an extended oxidative metabolism by CYP3A [66]. Genetic polymorphisms could partly explain inter and intra-patient variability of Indinavir pharmacokinetics since the data available remains controversial. It has been observed that $\mathrm{CYP} 3 \mathrm{~A} 5^{\star} 1$ carriers have significantly faster oral clearance than non-carriers. So far, no relationship was found between $\mathrm{CYP} 3 \mathrm{~A} 44^{\star} 1 \mathrm{~B}$ polymorphism and alterations in CYP3A substrate metabolism, but clinical data have shown an association between $\mathrm{CYP} 3 \mathrm{~A}^{\star} 1 \mathrm{~B}$ polymorphism and disease risk/treatment toxicity. Thus, therapeutic drug-monitoring appears to be a potent tool to achieve undetectable HIV-RNA and prevent Indinavir toxicity. In addition, as described for Atazanavir some may exhibit increased plasma levels of unconjugated bilirubin, which as 
Citation: Pesoa SA, Galván Bioch CA, Beltramo DM, Soria NW (2011) Human Immunodefieciency Virus: Pharmacogenetics of Antiretroviral Treatment. J Pharmacogenom Pharmacoproteomics S6:002. doi:10.4172/2153-0645.S6-002

mentioned above has been associated to competitive inhibition of UGT1A1.

Ritonavir: Dyslipidaemia is favored by a number of genetic and environmental determinants in the general population. Multiple genes, as well as important interactions among them, are involved in the development of the condition. In the field of HIV therapy, a recent study has found that Ritonavir related hypertriglyceridemia, a common side effect of the drug, is especially prevalent in patients with certain combinations of polymorphisms of genes ATP-binding cassette transporter A1 (ABCA1), apolipoproteína A5 (APOA5), apolipoproteína C3 (APOC3), apolipoproteína E (APOE), and cholesteryl ester transfer protein (CETP) [67].

\section{Fusion and entry inhibitors}

Maraviroc: Maraviroc is an oral CCR5 antagonist interfering with viral-cellular interactions at the entry process [68]. Considering the mechanism of action, this drug will be effective only in the subpopulation of HIV- 1-infected patients harboring viruses with selective tropism for the CCR5 receptor. The best-characterized human polymorphism observed in the CCR5 gene is the $\Delta 32$ deletion which associates with absence of cell surface-expressed CCR5 molecule. Subjects homozygous for this mutation $(\Delta 32 / \Delta 32)$ are highly resistant to HIV infection $[69,70]$ while heterozygous (WT/ $\Delta 32$ ) subjects often show slower disease progression rates [71]. Testing for $\Delta 32$ is recommended before starting Maraviroc based therapy.

\section{Conclusions and Future Perspectives}

Pharmacogenomics has provided the first clinically helpful results in the treatment of HIV infection, a field where toxicities and drugs interactions are particularly relevant. These findings contribute to the conviction that personalized design of antiretroviral drug regimens may be feasible in the near future. Present data include partial information on a limited number of genes and gene variants since a great extent of the research and clinical implications reported have applied a candidate gene approach. High throughput screening strategies simultaneously analyzing large number of candidate genes and gene variants represent a step forward, which has only just begun. This could be particularly significant in early drug development where such approaches could optimize drug efficacy and toxicity profiles in a given population therefore allowing not only to predict the efficacy and safety of new compounds but also to guide decision making in the discovery and development of new antiretroviral agents.

\section{Acknowledgements}

D. Beltramo is a Career Member of Consejo Nacional de Investigaciones Científicas y Técnicas (CONICET). This study was supported by a grant from the Laboratorio de Análisis Clínicos Especializados (LACE). We especially acknowledge Dr. Eduardo Fernández and Dr. Osvaldo Elbarcha for continuous encouragement and support. This work was supported by a grant from Universidad Católica de Córdoba.

\section{References}

1. Gazzard B (2005) Antiretroviral therapy for HIV: medical miracles do happen. Lancet 366: 346-347.

2. Tozzi V (2010) Pharmacogenetics of antiretrovirals. Antiviral Res 85: 190-200.

3. Hammer SM, Eron JJ, Reiss P, Schooley RT, Thompson MA, et al. (2008) Antiretroviral treatment of adult HIV infection: 2008 recommendations of the International AIDS Society-USA panel. JAMA 300: 555-570.

4. d'arminio Monforte A, Cozzi-Lepri A, Phillips A, De Luca A, Murri R, et al. (2005)
Interruption of highly active antiretroviral therapy in HIV clinical practice: results from the Italian Cohort of Antiretroviral-Naive Patients. J Acquir Immune Defic Syndr 38: 407-416.

5. Rawlins MD (1981) Clinical pharmacology. Adverse reactions to drugs. Br Med $\mathrm{J}$ (Clin Res Ed) 282: 974-976.

6. Pirmohamed M, James S, Meakin S, Green C, Scott AK, et al. ( 2004) Adverse drug reactions as cause of admission to hospital: prospective analysis of 18 820 patients. BMJ 329: 15-19.

7. Rodriguez-Novoa S, Barreiro P, Jimenez-Nacher I, Soriano V (2006) Overview of the pharmacogenetics of HIV therapy. Pharmacogenomics J 6: 234-245.

8. Phillips EJ, Mallal SA (2008) Pharmacogenetics and the potential for the individualization of antiretroviral therapy. Curr Opin Infect Dis 21: 16-24.

9. Reynolds GP (2007) The impact of pharmacogenetics on the development and use of antipsychotic drugs. Drug Discov Today 12: 953-959.

10. Gumbo T (2008) Integrating pharmacokinetics, pharmacodynamics and pharmacogenomics to predict outcomes in antibacterial therapy. Curr Opin Drug Discov Devel 11: 32-42.

11. Ingelman-Sundberg $M(2008)$ Pharmacogenomic biomarkers for prediction of severe adverse drug reactions. N Engl J Med 358: 637-639.

12. Tozzi V, Libertone R, Liuzzi G (2008) HIV pharmacogenetics in clinical practice: recent achievements and future challenges. Curr HIV Res 6: 544-554.

13. Fellay J, Boubaker K, Ledergerber B, Bernasconi E, Furrer H, et al. (2001) Prevalence of adverse events associated with potent antiretroviral treatment: Swiss HIV Cohort Study. Lancet 358: 1322-1327.

14. Lamba J, Lamba V, Schuetz E (2005) Genetic variants of PXR (NR1/2) and CAR (NR1/3) and their implications in drug metabolism and pharmacogenetics. Curr Drug Metab 6: 369-383.

15. Dorr P, Westby M, Dobbs S, Griffin P, Irvine B, et al. (2005) Maraviroc (UK 427,857 ), a potent, orally bioavailable, and selective small-molecule inhibitor of chemokine receptor CCR5 with broad-spectrum anti-human immunodeficiency virus type 1 activity. Antimicrob Agents Chemother 49: 4721-4732.

16. Lazzarin A (2005) Enfuvirtide: the first HIV fusion inhibitor. Expert Opin Pharmacother 6: 453-464.

17. Kuritzkes DR, Jacobson J, Powderly WG, Godofsky E, DeJesus E, et al. (2004) Antiretroviral activity of the anti-CD4 monoclonal antibody TNX-355 in patients infected with HIV type 1. J Infect Dis 189: 286-291.

18. Tagat JR, McCombie SW, Nazareno D, Labroli MA, Xiao Y, et al. (2004) Piperazine-based CCR5 antagonists as HIV-1 inhibitors. IV. Discovery of 1-[(4,6-dimethyl-5-pyrimidinyl)carbonyl]- 4-[4-[2-methoxy-1(R)-4-(trifluoromethyl)phenyl] ethyl-3(S)-methyl-1-piperazinyl]- 4-methylpiperidine (Sch-417690/Sch-D), potent, highly selective, and orally bioavailable CCR5 antagonist. J Med Chem 47: 2405-2408

19. Khatib N, Das S (2010) PRO 140--a novel CCR5 co-receptor inhibitor. Recen Pat Antiinfect Drug Discov 5: 18-22.

20. Zhou N, Luo Z, Luo J, Liu D, Hall JW, et al. (2001) Structural and functional characterization of human CXCR4 as a chemokine receptor and HIV-1 coreceptor by mutagenesis and molecular modeling studies. J Biol Chem 276 42826-42833.

21. Buckheit RW, Watson K, Fliakas-Boltz V, Russell J, Loftus TL, et al. (2001) SJ-3366, a unique and highly potent nonnucleoside reverse transcriptase inhibitor of human immunodeficiency virus type 1 (HIV-1) that also inhibits HIV2. Antimicrob Agents Chemother 45: 393-400.

22. Ono M, Wada $Y$, Wu $Y$, Nemori R, Jinbo $Y$, et al. (1997) FP-21399 blocks HIV envelope protein-mediated membrane fusion and concentrates in lymph nodes. Nat Biotechnol 15: 343-348.

23. Cressey TR, Lallemant M (2007) Pharmacogenetics of antiretroviral drugs fo the treatment of HIV-infected patients: an update. Infect Genet Evol 7: 333-342.

24. Autar RS, Ananworanich J, Apateerapong W, Sankote J, Hill A, et al. (2004) Pharmacokinetic study of saquinavir hard gel caps/ritonavir in HIV-1-infected patients: $1600 / 100 \mathrm{mg}$ once-daily compared with $2000 / 100 \mathrm{mg}$ once-daily and 1000/100 mg twice-daily. J Antimicrob Chemother 54: 785-790. 
Citation: Pesoa SA, Galván Bioch CA, Beltramo DM, Soria NW (2011) Human Immunodefieciency Virus: Pharmacogenetics of Antiretroviral Treatment. J Pharmacogenom Pharmacoproteomics S6:002. doi:10.4172/2153-0645.S6-002

25. Pfister M, Labbé L, Hammer SM, Mellors J, Bennett KK, et al. (2003) Population pharmacokinetics and pharmacodynamics of efavirenz, nelfinavir, and indinavir: Adult AIDS Clinical Trial Group Study 398. Antimicrob Agents Chemother 47: 130-137.

26. Haas DW, Smeaton LM, Shafer RW, Robbins GK, Morse GD, et al. (2005) Pharmacogenetics of long-term responses to antiretroviral regimens containing Efavirenz and/or Nelfinavir: an Adult Aids Clinical Trials Group Study. J Infect Dis 192: 1931-1942.

27. Owen A, Pirmohamed M, Khoo SH, Back DJ (2006) Pharmacogenetics of HIV therapy. Pharmacogenet Genomics 16: 693-703.

28. Mangano A, Theiler G, Sala L, Capucchio M, Fainboim L, et al. (2001) Distribution of CCR5-Delta 32 and CCR2-64I alleles in an Argentine Amerindian population. Tissue Antigens 58: 99-102.

29. Motta P, Cibulsky L, lliovich E, Habegger de Sorrentino A (2000) [Frequency of the mutated allele of CCR -5 receptor in HIV-1 positive and negative individuals in the Province of Chaco]. Medicina (B Aires) 60: 431-434.

30. Biti R, Ffrench R, Young J, Bennetts B, Stewart G, et al. (1997) HIV-1 infection in an individual homozygous for the CCR5 deletion allele. Nat Med 3: 252-253.

31. Martinson JJ, Chapman NH, Rees DC, Liu YT, Clegg JB (1997) Global distribution of the CCR5 gene 32-basepair deletion. Nat Genet 16: 100-103.

32. Guerini FR, Delbue S, Zanzottera M, Agliardi C, Saresella M, et al. (2008) Analysis of CCR5, CCR2, SDF1 and RANTES gene polymorphisms in subjects with HIV-related PML and not determined leukoencephalopathy. Biomed Pharmacother 62: 26-30.

33. Galvan CA, Elbarcha OC, Fernandez EJ, Beltramo DM, Soria NW (2011) Distribution of Polymorphisms in Cytochrome P450 2B6, Histocompatibility Complex P5, Chemokine Coreceptor 5, and Interleukin 28B Genes in Inhabitants from the Central Area of Argentina. Genet Test Mol Biomarkers.

34. Marsh S, McLeod HL (2006) Pharmacogenomics: from bedside to clinical practice. Hum Mol Genet 15: 89-93.

35. Phillips KA, Veenstra DL, Oren E, Lee JK, Sadee W (2001) Potential role of pharmacogenomics in reducing adverse drug reactions: a systematic review. JAMA 286: 2270-2279.

36. van Leeuwen R, Lange JM, Hussey EK, Donn KH, Hall ST, et al. (1992) The safety and pharmacokinetics of a reverse transcriptase inhibitor, 3TC, in patients with HIV infection: a phase I study. AIDS 6: 1471-1475.

37. Robbins BL, Srinivas RV, Kim C, Bischofberger N, Fridland A (1998) Anti-human immunodeficiency virus activity and cellular metabolism of a potential prodrug of the acyclic nucleoside phosphonate 9-R-(2-phosphonomethoxypropyl) adenine (PMPA), Bis(isopropyloxymethylcarbonyl)PMPA. Antimicrob Agents Chemother 42: 612-617.

38. Kallianpur AR, Hulgan T (2009) Pharmacogenetics of nucleoside reversetranscriptase inhibitor-associated peripheral neuropathy. Pharmacogenomics 10: 623-637

39. Escaut L, Liotier JY, Albengres E, Cheminot N, Vittecoq D (1999) Abacavir rechallenge has to be avoided in case of hypersensitivity reaction. AIDS 13: 1419-1420.

40. Mallal S, Nolan D, Witt C, Masel G, Martin AM, et al. (2002) Association between presence of HLA-B*5701, HLA-DR7, and HLA-DQ3 and hypersensitivity to HIV-1 reverse-transcriptase inhibitor abacavir. Lancet 359: 727-732.

41. Hughes DA, Vilar FJ, Ward CC, Alfirevic A, Park BK, et al. (2004) Costeffectiveness analysis of HLA $B^{*} 5701$ genotyping in preventing abacavir hypersensitivity. Pharmacogenetics 14: 335-342.

42. Mallal S, Phillips E, Carosi G, Molina JM, Workman C, et al. (2008) HLA-B*5701 screening for hypersensitivity to abacavir. N Engl J Med 358: 568-579.

43. Zucman D, Truchis P, Majerholc C, Stegman S, Caillat-Zucman S (2007) Prospective screening for human leukocyte antigen-B*5701 avoids abacavir hypersensitivity reaction in the ethnically mixed French HIV population. J Acquir Immune Defic Syndr 45: 1-3.

44. Rodríguez-Nóvoa S, Cuenca L, Morello J, Córdoba M, Blanco F, et al. (2010) Use of the HCP5 single nucleotide polymorphism to predict hypersensitivity reactions to abacavir: correlation with HLA-B*5701. J Antimicrob Chemother 65: 1567-1569.

45. Galvan CA, Elbarcha OC, Fernandez EJ, Beltramo DM, Soria NW (2011) Rapid HCP5 single-nucleotide polymorphism genotyping: a simple allele-specific PCR method for prediction of hypersensitivity reaction to Abacavir. Clin Chim Acta 412: 1382-1384.

46. Anderson PL, Lamba J, Aquilante CL, Schuetz E, Fletcher CV (2006) Pharmacogenetic characteristics of indinavir, zidovudine, and lamivudine therapy in HIV-infected adults: a pilot study. J Acquir Immune Defic Syndr 42 441-449.

47. Madeddu G, Bonfanti P, De Socio GV, Carradori S, Grosso C, et al. (2008) Tenofovir renal safety in HIV-infected patients: results from the SCOLTA Project. Biomed Pharmacother 62: 6-11.

48. Kiser JJ, Aquilante CL, Anderson PL, King TM, Carten ML, et al. (2008) Clinica and genetic determinants of intracellular tenofovir diphosphate concentrations in HIV-infected patients. J Acquir Immune Defic Syndr 47: 298-303.

49. Izzedine H, Hulot JS, Villard E, Goyenvalle C, Dominguez S, et al. (2006) Association between ABCC2 gene haplotypes and tenofovir-induced proximal tubulopathy. J Infect Dis 194: 1481-1491.

50. Imaoka T, Kusuhara H, Adachi M, Schuetz JD, Takeuchi K, et al. (2007) Functional involvement of multidrug resistance-associated protein 4 (MRP4/ ABCC4) in the renal elimination of the antiviral drugs adefovir and tenofovir. Mol Pharmacol 71: 619-627.

51. Yamanaka H, Gatanaga H, Kosalaraksa P, Matsuoka-Aizawa S, Takahashi T, et al. (2007) Novel mutation of human DNA polymerase gamma associated with mitochondrial toxicity induced by anti-HIV treatment. J Infect Dis 195: 1419-1425.

52. Lewis W (2007) Pharmacogenomics, toxicogenomics, and DNA polymerase gamma. J Infect Dis 195: 1399-1401.

53. Lewis W, Day BJ, Copeland WC (2003) Mitochondrial toxicity of NRTI antivira drugs: an integrated cellular perspective. Nat Rev Drug Discov 2: 812-822.

54. Baruffini E, Lodi T (2010) Construction and validation of a yeast mode system for studying in vivo the susceptibility to nucleoside analogues of DNA polymerase gamma allelic variants. Mitochondrion 10: 183-187.

55. Lichtenstein KA, Delaney KM, Armon C, Ward DJ, Moorman AC, et al. (2003) Incidence of and risk factors for lipoatrophy (abnormal fat loss) in ambulatory HIV-1-infected patients. J Acquir Immune Defic Syndr 32: 48-56.

56. Carr A, Samaras K, Thorisdottir A, Kaufmann GR, Chisholm DJ, et al. (1999) Diagnosis, prediction, and natural course of HIV-1 protease-inhibitor-associated lipodystrophy, hyperlipidaemia, and diabetes mellitus: a cohort study. Lancet 353: 2093-2099.

57. Ribaudo HJ, Haas DW, Tierney C, Kim RB, Wilkinson GR, et al. (2006) Pharmacogenetics of plasma efavirenz exposure after treatment discontinuation: an Adult AIDS Clinical Trials Group Study. Clin Infect Dis 42 401-407.

58. Rotger M, Colombo S, Furrer H, Bleiber G, Buclin T, et al. (2005) Influence of CYP2B6 polymorphism on plasma and intracellular concentrations and toxicity of efavirenz and nevirapine in HIV-infected patients. Pharmacogenet Genomics 15: $1-5$

59. Schipani A, Wyen C, Mahungu T, Hendra H, Egan D, et al. (2011) Integration of population pharmacokinetics and pharmacogenetics: an aid to optima nevirapine dose selection in HIV-infected individuals. J Antimicrob Chemother 66: 1332-1339.

60. Haas DW, Ribaudo HJ, Kim RB, Tierney C, Wilkinson GR, et al. (2004) Pharmacogenetics of efavirenz and central nervous system side effects: an Adult AIDS Clinical Trials Group study. AIDS 18: 2391-2400.

61. Mouly SJ, Matheny C, Paine MF, Smith G, Lamba J, et al. (2005) Variation in oral clearance of saquinavir is predicted by CYP3A5*1 genotype but not by enterocyte content of cytochrome P450 3A5. Clin Pharmacol Ther 78: 605618

62. Lakhman SS, Ma Q, Morse GD (2009) Pharmacogenomics of CYP3A considerations for HIV treatment. Pharmacogenomics 10: 1323-1339. 
Citation: Pesoa SA, Galván Bioch CA, Beltramo DM, Soria NW (2011) Human Immunodefieciency Virus: Pharmacogenetics of Antiretroviral Treatment. J Pharmacogenom Pharmacoproteomics S6:002. doi:10.4172/2153-0645.S6-002

63. Rodríguez-Nóvoa S, Martín-Carbonero L, Barreiro P, González-Pardo G, Jiménez-Nácher I, et al. (2007) Genetic factors influencing atazanavir plasma concentrations and the risk of severe hyperbilirubinemia. AIDS 21: 41-46.

64. Solas C, Lafeuillade A, Halfon P, Chadapaud S, Hittinger G, et al. (2003) Discrepancies between protease inhibitor concentrations and viral load in reservoirs and sanctuary sites in human immunodeficiency virus-infected patients. Antimicrob Agents Chemother 47: 238-243.

65. Bertrand J, Treluyer JM, Panhard X, Tran A, Auleley S, et al. (2009) Influence of pharmacogenetics on indinavir disposition and short-term response in HIV patients initiating HAART. Eur J Clin Pharmacol 65: 667-678.

66. Flexner C (1998) HIV-protease inhibitors. N Engl J Med 338: 1281-1292.

67. Arnedo M, Taffé P, Sahli R, Furrer H, Hirschel B, et al. (2007) Contribution of 20 single nucleotide polymorphisms of 13 genes to dyslipidemia associated with antiretroviral therapy. Pharmacogenet Genomics 17: 755-764.
68. Westby M, van der Ryst E (2005) CCR5 antagonists: host-targeted antivirals for the treatment of HIV infection. Antivir Chem Chemother 16: 339-354.

69. Liu R, Paxton WA, Choe S, Ceradini D, Martin SR, et al. (1996) Homozygous defect in HIV-1 coreceptor accounts for resistance of some multiply-exposed individuals to HIV-1 infection. Cell 86: 367-377.

70. Samson M, Libert F, Doranz BJ, Rucker J, Liesnard C, et al. (1996) Resistance to HIV-1 infection in caucasian individuals bearing mutant alleles of the CCR- 5 chemokine receptor gene. Nature 382: 722-725

71. Eugen-Olsen J, Iversen AK, Garred P, Koppelhus U, Pedersen C, et al. (1997) Heterozygosity for a deletion in the CKR- 5 gene leads to prolonged AIDSfree survival and slower CD4 T-cell decline in a cohort of HIV-seropositive individuals. AIDS 11: 305-310.

This article was originally published in a special issue,

Pharmacogenetics:HIV handled by Editor(s). Dr. Awewura Kwara, Brown

University, USA; Dr. Adrian L Lerena, University of Extremadura, Spain 